\title{
ESTUDIO DEL POLEN ATMOSFÉRICO Y SU RELACION CON LA VEGETACIÓN LOCAL. LA PLATA, ARGENTINA
}

\author{
Daniela S. NITIU \\ Cátedra de Palinología. Facultad de Ciencias Naturales y Museo. Institutos, Cátedras y Laboratorios. \\ Universidad Nacional de La Plata. Calle 64 N³. 1900. La Plata, Argentina. \\ danielanitiu@yahoo.com.ar
}

Recibido el 27 de abril de 2009, aceptado para su publicación el 9 de julio de 2009 Publicado "on line" en octubre de 2009

\begin{abstract}
RESUMEN. Estudio del polen atmosférico y su relación con la vegetación local. La Plata, Argentina. Se analizó el registro de polen en la atmósfera de la ciudad de La Plata $\left(34^{\circ} 55^{\prime} \mathrm{S}, 57^{\circ} 57^{\prime} \mathrm{W}^{\circ}\right)$ y su relación con la vegetación arbórea del área. La toma de muestras polínicas se llevó a cabo con un captador tipo Hirst (Lanzoni VPPS 2000) y se censó la vegetación arbórea de $1 \mathrm{~km}^{2}$. El 99\% del polen determinado se correspondió con fuentes emisoras locales. El 53\% de los taxa no estuvo representado en el registro dado, principalmente por su polinización entomófila. Se registró polen extralocal y, en menor escala, polen regional y extraregional. El índice de Representatividad de los 24 taxa expresados en el registro polínico permitió reagruparlos en las categorías, Subrepresentados a Aesculus hippocastanum L., Tilia moltkey L., Jacaranda mimosifolia D. Don., Robinia pseudoacacia L., Persea americana Mill., Ligustrum lucidum Aiton, Cedrus sp.pl. D.Don, Betula pendula Roth, Liquidambar styraciflua L., Ulmus sp.pl. L., Pinus sp.pl. L., Alnus acuminata Humb., Juglans sp. L., Populus deltoides L., Acer negundo L., Phoenix sp. Hort ex Chabaud., Fraxinus sp.pl. Tourn ex L. y Salix humboltiana L. Equirepresentados a Celtis sp. L., Morus sp. L. y Quercus sp. L. y Sobrerepresentados a Platanus sp. Mill. ex-Muenchh, Ricinus sp. L. y Cupressaceae Rico. ex-Bartl. El análisis polínico resulta adecuado principalmente para caracterizar a las especies de polinización anemófila del área, pero no siempre es representativo de la contribución de especies de polinización entomófila.
\end{abstract}

Palabras clave. Polen, atmósfera, vegetación urbana, La Plata, Argentina

ABSTRACT. Study of atmospheric pollen and its relationship with the local vegetation. La Plata, Argentina. The pollen record in the atmosphere of the City of La Plata $\left(34^{\circ} 55^{\prime} \mathrm{S}, 57^{\circ} 57^{\prime} \mathrm{W}\right)$ and its relationship with the arboreal vegetation in the area was analyzed. Sampling was carried out with a Hirst-type spore trap (Lanzoni VPPS 2000) and a census of the trees in $1 \mathrm{~km}^{2}$ was taked. $99 \%$ of the identified pollen corresponded to local emission sources. $53 \%$ of taxa was not represented in the record due mainly to entomophilous pollination. Extralocal pollen and, in a lesser extent, regional and extraregional pollen was registered. The Index of Representativeness of the 24 taxa expressed in the pollen record allowed to regroup them in 3 categories. Subrepresented: Aesculus hippocastanum L., Tilia moltkey L., Jacaranda mimosifolia D. Don., Robinia pseudoacacia L., Persea americana Mill., Ligustrum lucidum Aiton, Cedrus sp.pl. D.Don, Betula pendula Roth, Liquidambar styraciflua L., Ulmus sp. pl. L., Pinus sp.pl. L., Alnus acuminata Humb., Juglans sp. L., Populus deltoides L. , Acer negundo L., Phoenix sp. Hort ex Chabaud., Fraxinus sp.pl. Tourn ex L. and Salix humboltiana L. Equirepresented to Celtis sp. L., Morus sp. L. and Quercus sp. L. and Overrepresented: 
Platanus sp. Mill. ex-Muenchh, Ricinus sp. L. and Cupressaceae Rico. ex-Bartl. Pollen analysis is appropriate to characterize anemophilous species in the area, what not always is representative of the contribution of entomophilous pollinated species.

\section{INTRODUCCIÓN}

La composición de la vegetación de un área determina la diversidad y el número de tipos polínicos y su concentración en la atmósfera; sin embargo, el mecanismo de polinización, la ubicación y la densidad de las fuentes emisoras modifican directamente dicha relación. En general, el polen atmosférico es un indicador del inicio, desarrollo y máximo de las fases florales de la vegetación anemófila. En cambio, el final de la floración no está precisamente indicado por la curva polínica ya que los granos de polen permanecen en el aire después de que el polen ya ha sido emitido y las anteras se han marchitado (O'Rourke, 1990).

La capacidad de dispersión depende, en primer término, de las características de la planta originaria, ya que cada especie perece obedecer a un patrón que se refleja en el espectro polínico de una localidad, la cual varía con los días y con los años, así como de la noche al día. (Galán et al. 1991, Trigo et al. 1997).

El objetivo del trabajo fue analizar cualicuantitativamente la nube polínica de la ciudad de la Plata y el grado de representatividad de la vegetación local. Asimismo, tratar de interpretar la presencia en el registro de aquellos tipos polínicos provenientes de áreas alejadas al captador.

La ciudad de la Plata (34 $55^{\circ} \mathrm{S}$ and $\left.57^{\circ} 57^{\prime} \mathrm{W}\right)$ está localizada en el Noreste de la Provincia de Buenos Aires, Argentina en la Provincia Fitogeográfica Pampeana, Distrito Este (Cabrera, 1976). Presenta una vegetación arbórea muy abundante principalmente exótica distribuida en el arbolado urbano y en numerosos parques y jardines de la ciudad. El clima, de acuerdo a la clasificación de Thornthwaite
(Burgos \& Vidal, 1951) es mesotermal, subhúmedo/húmedo con marcada estacionalidad.

\section{MATERIAL Y MÉTODOS}

Se realizó un relevamiento de la vegetación arbórea del arbolado urbano de un área de $1 \mathrm{~km}^{2}$ desde el sitio de muestreo.

El monitoreo se realizó con un captador Lanzoni VPPS 2000 (Lanzoni Manufactiring Co., Italy) de recambio semanal. El mismo estuvo emplazado en una terraza de un edificio céntrico de la ciudad a 15 metros de altura. Las muestras fueron examinadas en un microscopio óptico con una magnificación de 200X usando cinco transectas horarias. La concentración se expresó en granos de polen $/ \mathrm{m}^{3}$ de aire (Galán et al. 2007).

La determinación de los granos de polen se basó en la bibliografía de referencia (Aira et al. 2005; Bassett et al. 1978; Ciampolini \& Cresti, 1991; Docampo Fernández, 2008; Moore et al. 1991) y la colección de referencia de la Cátedra de Palinología (Facultad de Ciencias Naturales y Museo, Universidad Nacional de La Plata, Argentina).

Los datos de concentración total se refieren a la suma total de polen emitido en el período de estudio.

Se aplicó a cada taxa el Índice de Representatividad (IR) de Heim (1970). (Caramiello et al. 1991; Latorre et al. 2001; Nitiu, 2008). El Índice muestra el grado en que la vegetación de un área está representada en la nube de polen. Dicho valor es un cociente entre el porcentaje de abundancia de cada taxa (V) respecto del total de la vegetación considerada $\mathrm{y}$ el porcentaje del polen $(\mathrm{P})$ de mismo taxa 
determinado en la atmósfera (V/P). A partir del valor del índice, los taxa fueron clasificados en las categorías: "sobrerepresentados" incluyendo a aquellos taxa cuyo valor del índice V/ $\mathrm{P}$ es inferior a 0.77 (polen $>$ vegetación); "equirepresentados" incluyen a aquellos taxa con un valor del índice V/P entre 1.26 y 0.77 y la categoría "subrepresentados" que incluye a aquellos taxa cuyo valor del índice V/P es mayor a 1.26 (vegetación>polen).

Se aplicó el Análisis de Correlación de Spearman con el objetivo de analizar la relación entre la concentración de polen presente en la nube y la vegetación emisora. Se calculó $r$ (coeficiente de correlación) para un grado de significación del 95\%.

\section{RESULTADOS}

\section{Análisis de la vegetación en relación al registro de polen}

Se determinaron 24 tipos polínicos en el registro cuyas fuentes emisoras fueron relevadas próximas al muestreador. El 84\% de ellos representa a taxa de polinización anemófila y el $16 \%$ a taxa de polinización entomófila (tab. 1).

En el muestreo no se detecta la presencia polínica de 27 taxa censados en el área. El $14.3 \%$ son taxa de polinización anemófila y el $85.7 \%$ de polinización entomófila (tab. 2).

Tipos polínicos como Casuarina, Castanea y Myrtaceae fueron identificados en el registro, hallándose sus fuentes emisoras en un área específico en los alrededores de la ciudad, lo cual destaca el aporte extralocal y la capacidad de dispersión de estos taxa.

A pesar de ser entomófilos, se determinaron escasos granos de polen de Calliandra, Mimosa, Schinus y Strelitzia, cuya procedencia es de vegetación muy cercana plantada en jardines de casas.

Se registró polen regional de Olea, Juncus y Ephedra, cuyos taxa están localizados a

\begin{tabular}{cl}
\hline $\begin{array}{c}\text { Número de } \\
\text { individuos }\end{array}$ & \multicolumn{1}{c}{ Taxa } \\
\hline 1020 & Fraxinus sp pl. \\
736 & Platanus acerifolia \\
420 & Tilia moltkei \\
211 & Cupressacea \\
98 & Acer negundo \\
75 & Jacaranda mimosiifolia \\
59 & Cedrus spp. \\
54 & Celtis australis \\
45 & Aesculus hippocastanum \\
34 & Ulmus spp. \\
26 & Morus alba \\
22 & Robinia pseudoacacia \\
20 & Populus deltoides \\
16 & Pinus spp. \\
15 & Phoenix sp. \\
9 & Quercus robur \\
8 & Ligustrum lucidum \\
8 & Salix humboldtiana \\
7 & Liquidambar styraciflua \\
6 & Juglans australis \\
2 & Betula pendula \\
2 & Ricinus communis \\
1 & Alnus acuminata \\
1 & Persea americana \\
\hline
\end{tabular}

Tabla 1: Lista de taxa y número de ejemplares censados en el área de estudio cuyos tipos polínicos han sido identificados y determinados en la nube de polen. List of taxa and number of tree in the area of study whose pollen types have been identified and determined in the cloud of pollen.

pocos kilómetros de la ciudad sobre las costas del Río de la Plata. También se registró polen extraregional de Nothofagus proveniente de los Andes subandinos (Argentina), a $1600 \mathrm{~km}$ al SW de la ciudad de La Plata.

\section{Representatividad de los taxa en el registro de polen}

Se analizó el índice de Representatividad con los 24 taxa presentes en el registro de polen. Los taxa subrepresentados fueron Aesculus hippocastanum L., Tilia moltkey L., Jacaranda mimosifolia D. Don., Robinia pseudoacacia L., Persea americana Mill., Ligustrum lucidum Aiton, Cedrus sp.pl. D.Don, Betula pendula 


\begin{tabular}{cl}
\hline $\begin{array}{c}\text { Número de } \\
\text { individuos }\end{array}$ & \multicolumn{1}{c}{ Taxa } \\
\hline 56 & Tipuana tipu \\
36 & Magnolia grandiflora \\
34 & Citrus aurantium \\
22 & Hovenia dulcis \\
20 & Styphnolobium japonicum \\
19 & Prunus cerasifera var. atropurpurea \\
13 & Ailanthus altissima \\
13 & Erythrina crista-galli \\
12 & Gleditsia triacanthos \\
10 & Araucaria spp. \\
6 & Lagerstroemia indica \\
5 & Bauhinia forficata subsp. pruinosa \\
3 & Cryptomeria japonica \\
3 & Chorisia insignis \\
3 & Ficus elastica \\
3 & Sterculia platanifolia \\
2 & Brachychiton populneum \\
2 & Feijoa sellowiana \\
2 & Livistona chinensis \\
2 & Melia azederach \\
2 & Syagrus spp. \\
1 & Abies alba \\
1 & Cinnamomum glanduliferum \\
1 & Eriobotrya japonica \\
1 & Grevillea robusta \\
1 & Hibiscus mutabilis \\
1 & Phytolacca dioica \\
&
\end{tabular}

Tabla 2: Lista de taxa y número de ejemplares encontrados en el área, cuyos tipos polínicos no han sido identificados en el muestreo aerobiológico. List of taxa and number of trees found in the area, whose pollen types have not been identified in the aerobiológico sampling.

Roth, Liquidambar styraciflua L., Ulmus sp.pl. L., Pinus sp.pl. L., Alnus acuminata Humb., Juglans sp. L., Populus deltoides L. , Acer negundo L., Phoenix sp. Hort ex Chabaud., Fraxinus sp.pl. Tourn ex L. y Salix humboltiana L. (fig. 1).

La categoría "equirepresentado" $(<1.26$ $\mathrm{V} / \mathrm{P}>0.77$ ) significó el $12.5 \%$ con tres taxones. Ellos fueron Celtis sp. L., Morus sp. L. y Quercus sp. L. (fig. 1).

La categoría "sobrerepresentado" (V/P $<0.77$ ) significó el $12.5 \%$ con tres taxones. Ellos fueron Platanus sp. Mill. ex-Muenchh, Ricinus sp. L. y Cupressaceae Rico. ex-Bartl (fig. 1).

\section{Análisis estadístico}

Se realizó un análisis de correlación entre el número total de ejemplares de cada taxón y la concentración total de polen emitida por los mismos en el periodo de muestreo. En la figura 2 puede observarse un alto nivel de significación $(\mathrm{r}=0.822, \mathrm{p}=0.011)$ con el $87.5 \%$ de los taxa incluidos en el intervalo de confianza. Los taxa fuera del mismo, son Cupressaceae y Platanus sp. que se hallan por encima de la línea recta y Tilia por debajo de la misma.

Esta observación, es un primer indicio de que existe una representatividad diferencial entre los taxones en el espectro polínico, y que se podría deber al tipo de estrategia reproductiva señalada por sus especies, siendo Cupressaceae y Platanus de polinización anemófila y Tilia sp. de polinización entomófila.

En una segunda instancia, el análisis de correlación fue realizado con los 18 taxa del grupo subrepresentado (fig. 1) y mostró un alto nivel de significación $(\mathrm{r}=0.921, \mathrm{p}$ $=1.425^{*} 10^{-7}$ ) (fig. 3). El diagrama expone que el $94.4 \%$ de los taxa están incluidos en el intervalo de confianza, muy próximos a la línea de regresión y a la ordenada de origen. Cinco de estos taxa presentan características entomófilas y son Aesculus sp., Tilia sp., Jacaranda sp.., Robinia sp., Persea sp. y Ligustrum sp. Y diez de ellos muestran características anemófilas y son: Cedrus sp., Betula sp., Liquidambar sp., Ulmus sp., Pinus sp., Alnus sp., Juglans sp., Populus sp., Acer sp., Phoenix sp, Fraxinus sp. y Salix sp.

Tilia está representado por T. molkey en la vegetación y se ubica en el tercer lugar respecto del total censado con el 13.3\% (tab. 1), aunque registró una muy baja cantidad de polen en la atmósfera en los tres años.

Fraxinus sp. es el taxon más abundante de la vegetación urbana (32.4\%) (tab. 1) representado por $F$. americana L. y F. excelsior var. aurea L. y respecto al total anual de polen, es segundo en los tres años de monitoreo (Nitiu, 2006; Nitiu \& Romero, 2001). 


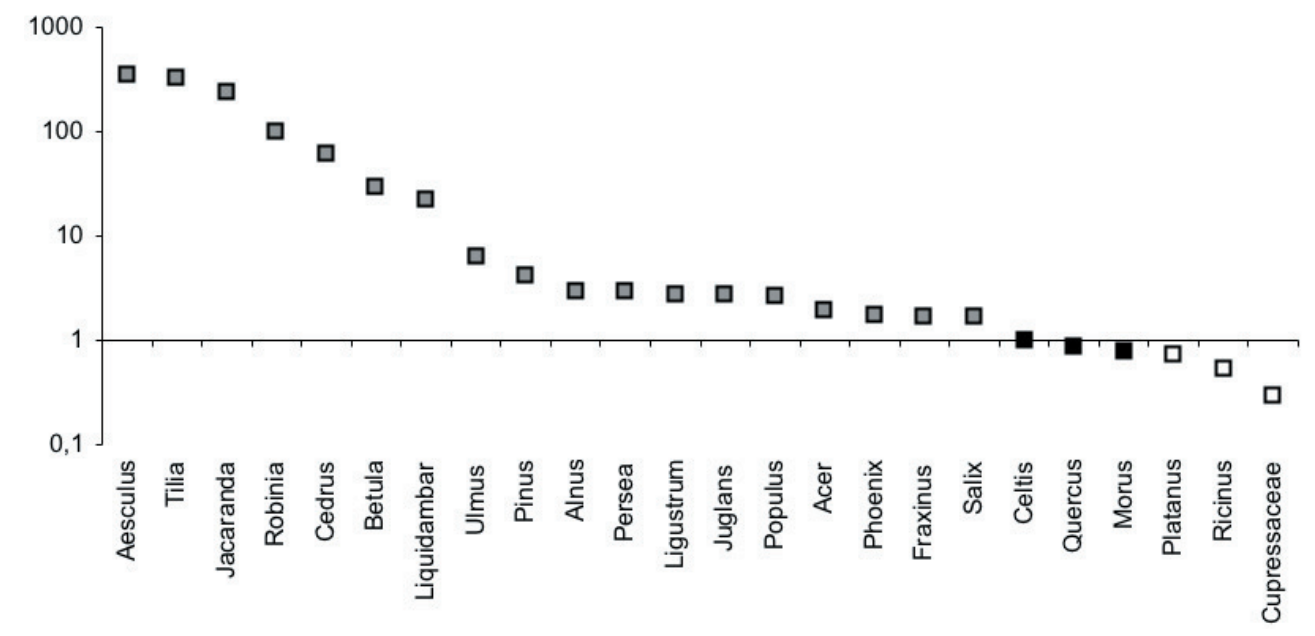

Figura 1: Índice de Representatividad analizado con los taxa cuyos granos de polen estuvieron presentes en el registro. Los cuadrados en gris indican los taxa subrepresentados, los cuadrados en negro indican los taxa equirepresentados y los cuadrados en blanco los taxa sobrerepresentados. Representativity Index with the taxa whose pollen grains were present in the register. The grey squares indicate the underrepresented taxa, black squares indicate the taxa equirepresented and blank squares sobrerepresented taxa.

Acer sp. se halló en el intervalo de confianza del 95\% próximo al límite inferior de la línea con valores muy bajos de ambos coeficientes. Se halla representado en el área por $A$. negundo L. y en el quinto lugar con el $3.4 \%$ respecto al total de la vegetación censada (tab. 1).

Los quince taxones restantes se ubicaron dentro del intervalo de confianza muy próximos o sobre la línea y a su vez muy cercanos a la ordenada al origen. De ellos, cinco taxones presentaron características entomófilas y fueron Aesculus sp., Jacaranda sp., Robinia sp., Persea sp. y Ligustrum sp. Por otra parte, los otros diez taxones presentaron características anemófilas y fueron Cedrus sp., Liquidambar sp., Betula sp., Alnus sp., Populus sp., Ulmus sp., Juglans sp., Pinus sp., Phoenix sp. y Salix sp.

El análisis de correlación del grupo equirepresentado (fig. 1) muestra un alto grado de significación $(\mathrm{r}=0.985, \mathrm{p}=0.110)$ (fig. 4). En este grupo, 3 taxa: Celtis sp., Morus sp. y Quercus sp. fueron los destacados. Ellos están dentro del intervalo de confianza y muy próximos a la línea a diferente distancia del origen.

Celtis está presente en la ciudad con $C$. australis L. en zonas cercanas al muestreador, principalmente en zonas de parquizadas aportando el $1.8 \%$ al total censado (tab. 1) (Nitiu, 2003a).

Morus esta presente mayoritariamente en el arbolado urbano $(0.8 \%)$ (tab. 1) con la especie M. alba. L. (Nitiu, 2003b).

Quercus es un género anemófilo el cual está representado en la vegetación del área por Q. robur. L. en muy bajo número de ejemplares (tab. 1).

Con respecto al grupo sobrerepresentado (fig. 1), el análisis mostró buena dependencia entre ambas coordenadas y registra todos los taxones dentro del intervalo de confianza del $95 \%(\mathrm{r}=0.915, \mathrm{p}=0.264)$ (fig. 5).

Platanus registró el valor más alto de los coeficientes. Está presente en la vegetación por $P$. acerifolia, ubicándose en segundo lugar (24\%) en el censo (tab. 1). Además registra la máxima concentración (Nitiu \& Mallo, 2002; Nitiu \& Romero, 2002) aportada al total del 


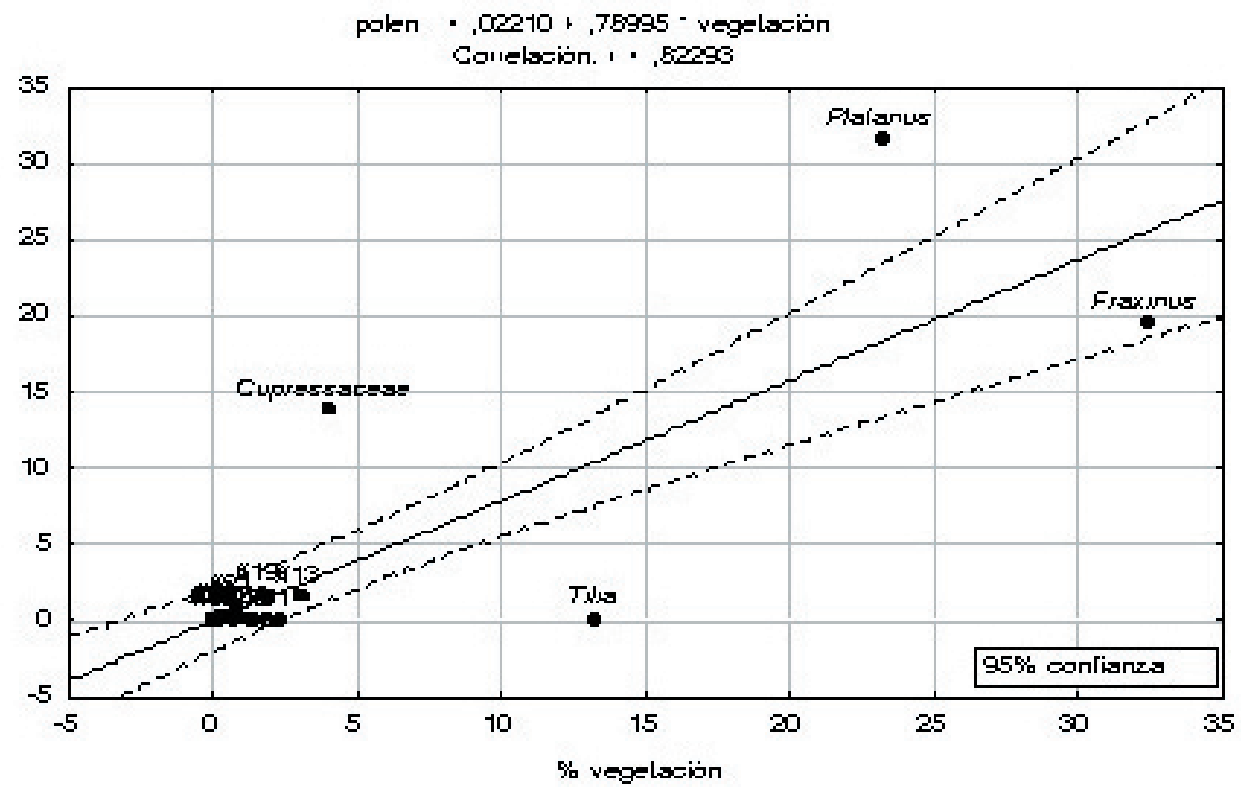

Figura 2: Análisis de correlación con todos los taxa analizados en el área. Analysis of correlation with all taxa analysed in the area.

muestreo.

Cupressaceae estuvo representada por Thuja sp.pl. L. y Juniperus sp.pl. L. y mayoritariamente por Cupressus sp.pl. L., significando el $7.2 \%$ de la vegetación total (tab. 1). La contribución polínica anual de esta familia en la atmósfera es muy alta y se encuentra en tercer lugar respecto del polen total anual (Nitiu, 2008). Sus géneros presentan polinización anemófila con un desarrollo importante y simultáneo de las fases florales (Caramiello et al., 1991).

\section{DISCUSIÓN Y CONCLUSIÓN}

El 47\% de los taxones arbóreos presentes en la vegetación urbana censada estuvieron representados en el registro polínico. Esto se asocia al elevado número de especies de polinización anemófila siendo las más abundantes Fraxinus sp., Platanus sp. y
Cupressaceae, como así también las de mayor aporte polínico.

Por otra parte, el 53\% de los taxones arbóreos censados no estuvieron representados en el espectro polínico. Este valor expone la presencia de taxones entomófilos que ornamentan parques y calles cercanos al área de muestreo que presentan además un número relativamente alto de plantas. Tipuana tipu (Benth.) Kuntze, Magnolia grandiflora L. y Citrus aurantium L. (tab. 2) pertenecen a esta categoría entre otros, cuyos rasgos en las estructuras reproductivas dificultan la difusión y transporte del polen por el aire.

La ausencia de ciertos pólenes anemófilos en los registros se deben a diversos factores particulares de este muestreo y que están directamente relacionados con la velocidad del viento, turbulencia y la altura del suelo en la que se encuentran las anteras y inversa a la vegetación que emite o a obstáculos que pueden producirse (D'Antoni, 2008) como en 


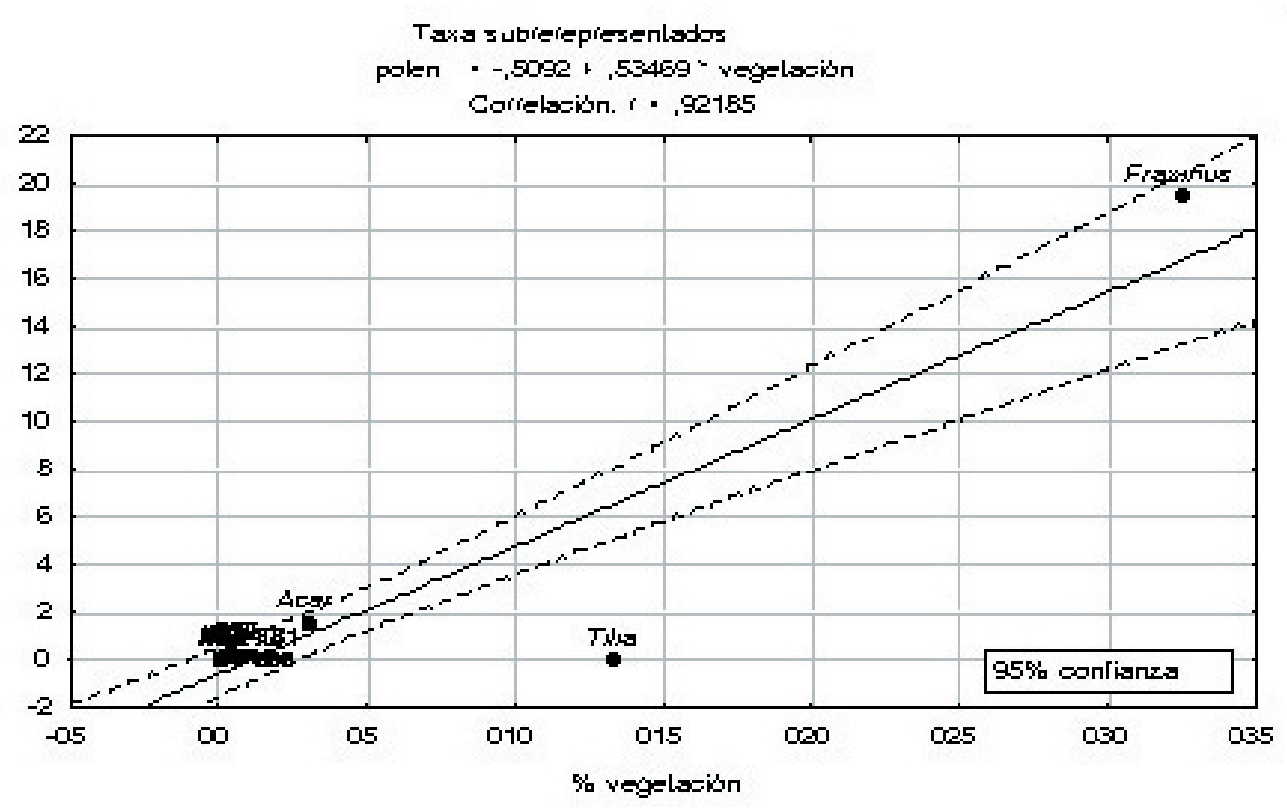

Figura 3: Análisis de correlación para los dieciocho taxa del grupo subrepresentado. Analysis of correlation to the eighteen taxa of the subrepresented group.

el caso de Syagrus Mart. cuya ausencia se debe principalmente a la presencia de edificaciones elevadas a su alrededor. Asimismo, la planta censada de Araucaria sp.pl. Juss. y Abies alba Mill. se corresponden con ejemplares femeninos y la ausencia de polen de Cryptomeria japonica (L.F.) D.Don. se debe a que la planta presenta un estado fenológico jóven sin desarrollo aún de las estructuras reproductivas (tab. 2).

El $99 \%$ de los tipos polínicos registrados en la atmósfera, provinieron de fuentes locales y extralocales, coincidiendo con lo ocurrido en otras ciudades del país como Mar del Plata (Latorre, 1997), Tucumán (García, 2006), Buenos Aires (Nitiu et al, 2003). Esta información es de gran interés clínico ya que, en genera, la mayoría de estas partículas está asociada a la aparición de transtornos alérgicos, en especial en las vías respiratorias de la población.

El aporte regional de Olea, Juncus y Ephedra, desde la zona costera del Río de La
Plata al este de la ciudad pone en evidencia la importancia del factor dirección y velocidad del viento predominante en esa área, dado que dicha vegetación es característica y muy abundante en la zona, estando ausente en la ciudad (Majas \& Romero, 1992; Nitiu et al., 2003).

La fuente de emisión de polen más alejada del sitio de muestreo, correspondió a Nothofagus que representa a una vegetación ubicada a más de $1000 \mathrm{~km}$ del punto de muestreo. Esta presencia estaría dada por las características propicias del grano de polen para mantenerse en las capas bajas de la atmósfera y ser transportado por las corrientes de vientos provenientes de los bosques subandinos del SW argentino, a través de corrientes convectivas (Gassmann \& Perez, 2006). Este tipo de polen también ha sido citado para las ciudades de Buenos Aires (Nitiu et al., 2003) y en Mar del Plata por Latorre (1997) y Pérez et al. (2001).

La ciudad de La Plata presenta una cuantiosa y diversa vegetación arbórea 


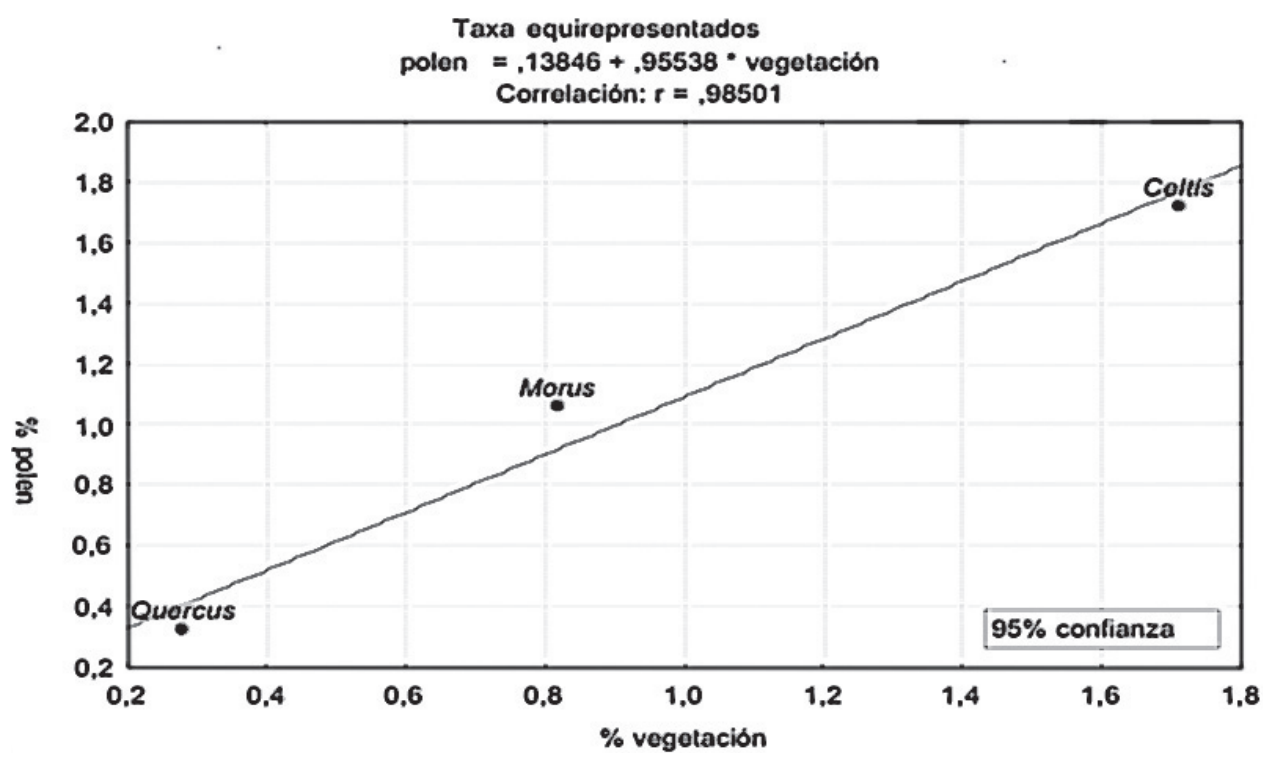

Figura 4: Análisis de correlación para los taxa del grupo equirepresentado. Analysis of correlation to the taxa of the equirepresented group.

distribuida en sus casi 20 plazas, 5 parques y numerosos jardines como así también en el arbolado urbano de calles y ramblas.

Es rasgo característico que la representación polínica en la atmósfera de cada taxón está asociada principalmente a la cercanía de la fuente emisora al captador, sin embargo el factor abundancia en el área, grado de madurez de los ejemplares y alta productividad polínica juegan un rol asociado muy importante. Así se pudieron evidenciar tres categorías de representatividad taxonómica en el registro polínico.

La mayor subrepresentación estuvo dada por Aesculus sp. (fig. 1) que pone en evidencia un alto numero de ejemplares en la vegetación y muy escasa en el registro de polen. Según Pla Dalmau (1961), estos granos de polen poseen características anemófilas en su tamaño y escultura y características entomófilas por una baja productividad de polen y su dispersión es por insectos.

Otro taxa de este grupo fue Tilia sp. el cual presenta polinización entomófila y su presencia en el aire fue casi nula, no obstante es el tercer taxón más representado en la vegetación urbana y muy abundante en el área de muestreo como se pudo observar en las gráficas. Tal diferencia se debería principalmente a que corresponde a una especie entomófila e híbrida cuyas estructuras de reproducción no suelen alcanzar el estado óptimo para la fertilización, así como también a la atrofia o deformidad en sus granos de polen, características observadas en el muestreo. (Faegri \& Iversen, 1989; Spieksma et al. 1994).

Se destacan también Ulmus sp. y Populus sp. cuya densidad arbórea es moderada en el área y principalmente son taxa de arbolado de calles. A pesar de ser géneros estrictamente anemófilos, Ulmus sp., aunque es un alto productor de polen (Solomon \& Durham, 1967), presenta granos con una pared muy gruesa y un tamaño relativamente grande, condiciones éstas que le dificultarían la dispersión. Por su parte, Populus sp., a pesar de tener una gran 


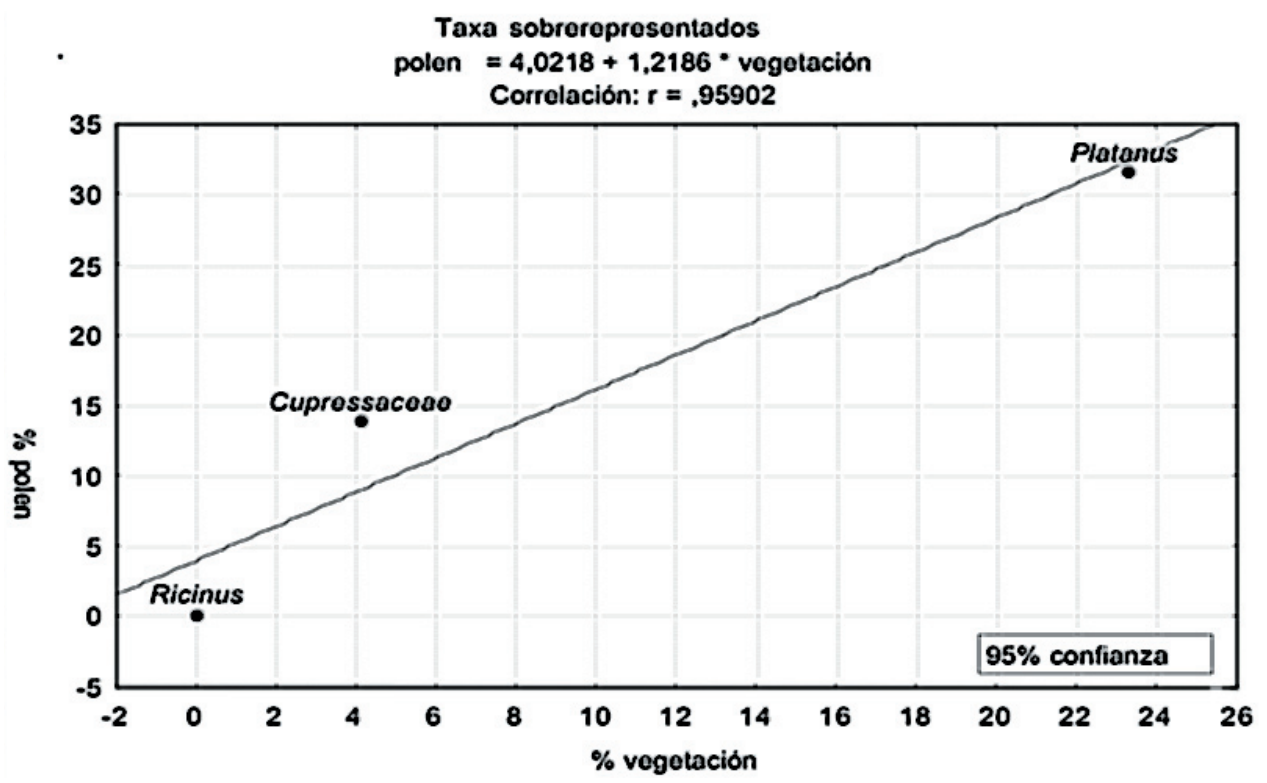

Figura 5: Análisis de correlación para los taxa del grupo sobrerepresentado. Analysis of correlation to the taxa of the sobrerepresented group.

productividad y sus granos de polen ser muy livianos, el grado de dispersión es acotado a su fuente de emisión.

Fraxinus sp. fue el taxón más abundante de la vegetación urbana y se encontró masivamente en el sector de muestreo. Pese a ello su concentración en la atmósfera no fue los suficientemente representativa de ello. Es considerado un género de baja productividad por Andersen, (1973) y Jackson \& Wong, (1994), lo que explicaría en parte estos resultados.

Celtis sp., Morus sp. y Quercus sp. se hallaron equirepresentados en diverso grado en el registro de polen, pero con la particularidad de presentar aportes de concentración a la atmósfera que se los puede vincular al número de plantas presentes en el área. Son géneros que se consideran anemófilos con alta productividad de polen; sin embargo la superficie rugosa y la pared gruesa de los granos de polen de Quercus le confieren cierta característica entomófila (Käpylä, 1984).
La sobrerepresentación de Platanus sp. sugiere que fue debida a su alta densidad en el arbolado como en los parques censados. Asimismo el porte y la dimensión de las plantas influyen en la producción polínica y la elevada concentración aportada por este taxón en el registro de polen, (Cariñanos et al., 2004).

Cupressaceae, está conformado por plantas anemófilas de los géneros Thuja sp. y Cupressus sp. principalmente, con numerosas fuentes de emisión cuya elevada productividad polínica y rasgos aerodinámicos de los granos, corroborados por los altos valores de concentración atmosférica registrados en el muestreo.

El espectro de polen atmosférico de la ciudad reflejó en gran parte la composición de la vegetación circundante y su abundancia en el área de estudio. Sin embargo, el tipo de polinización de los taxa, las características aerodinámicas de los granos de polen, la distancias entre la fuente emisora y la fuente colectora juegan un rol muy importante en el 
momento de determinar la representatividad polínica de la vegetación.

AGRADECIMIENTOS. Este trabajo de investigación se ha hecho posible gracias al subsidio de investigación otorgado por CONICET (Comisión Nacional de Investigaciones Científicas y Tecnológicas), así como el apoyo económico de la Universidad Nacional de la Plata (UNLP).

\section{REFERENCIAS}

AIRA, M. J., V. JATO e I. IGLESIAS -2005-Calidad del aire. Polen y esporas en la Comunidad Gallega. Eds. Xunta de Galicia. Consellería de Medio Ambiente.

ANDERSEN, S. TH. -1973-. The differential pollen productivity of trees and its significance for the interpretation of a pollen diagram from a forested region. En Quaternary plant ecology. Eds. H.J.B. Birks and R.G. West. Blackwell, Oxford.

BASSETT, I. J., C.W. CROMPTON \& J.A. PARLELEE -1978-. An atlas of airborne pollen grains and common spores of Canada. Monogr. 18. Ottawa. Canadian Department of Agriculture, Ottawa.

BURGOS, J. J. \& A. L. VIDAL. -1951-. Los climas de la República Argentina según la nueva clasificación de Thornthwaite. Dir. Gen. Del S.M.N. Ser. Agrometeorol. Public. 3, 3-32. Buenos Aires.

CABRERA, A. L. -1976-. Regiones Fitogeográficas Argentinas. En Enciclopedia Argentina de Agricultura y Jardinería, Tomo II. Fascículo I. Editorial. ACME S.A.C.I., Buenos Aires, Argentina.

CARAMIELLO, R., C. SINISCALCO \& R. PIERVITTORI. -1991-. The relationship between vegetation and pollen depositation in soil and in biological trap. Grana 30, 291-230.

CARIÑANOS, P., C. GALÁN, P. ALCÁZAR \& E. DOMÍNGUEZ. -2004-. Analysis of the Particles transported with Dust-Clouds Cordoba, Southwestern Spain. Arch. Environ. Contam. Toxicol. 46: 141-146.

CIAMPOLINI, F. \& M. CRESTI. -1991-. Atlante dei principali pollini allergenici presenti in Italia.
Universitá di Siena.

D'ANTONI, H. -2008-. Arqueoecología: sistemática y caótica. Editorial CSIC. Madrid, España.

DOCAMPO FERNÁNDEZ, S. -2008-. Estudio aerobiológico de la atmósfera de la costa oriental de Málaga (sur de España) e incidencia de las esporas fúngicas en el interior de la cueva de Nerja. Tesis doctoral. Facultad de Ciencias. Departamento de Biología Vegetal. Málaga.

FÆGRI, K. \& IVERSEN, J. -1989- Textbook of pollen analysis. 4 th ed. by K. Fægri, P.E.

GALÁN, C., R. TORMO, J. CUEVAS, F. INFANTE \& E. DOMÍNGUEZ. -1991-. Theoretical daily variation patterns of airborne pollen in the South West of Spain. Grana 30: 201-209.

GALÁN, C., P. CARIÑANOS, P. ALCÁZAR TENO y E. DOMÍNGUEZ VILCHES. -2007-. Manual de Calidad y Gestión de la Red Española de Aerobiología, REA. Servicio de Publicaciones de la Universidad de Córdoba. España.

GARCÍA, M. E. -2006-. Lluvia polínica en Selvas Montanas de la provincia de Tucumán (Argentina). Revista del Museo Argentino de Ciencias Naturales Vol. VIII (2):159-164.

GASSMANN, M. I. \& C. PEREZ. -2006-. Trayectories associated to regional and extraregional pollen transport in the southeast of Buenos Aires province, Mar del Plata (Argentina). Int J. Biometeorol 50: 280-291.

HEIM, J. -1970-. Les relations entre les espectres polliniques récents et la végétation actuelle en Europe occidentale. Mem. $\mathrm{n}^{\circ} 4$ de la Soc. Roy. Bot. Belg, 181 p. En: C. Montanari, M.A. Guido y M. Bottero, 1986. La pioggia pollinica rilevata al suolo nellárea urbana di Geneva. Archivo Botanico e Biogeográfico Italiano 62: 146-174.

JACKSON, S. T. \& A. WONG. -1994-. Using forest patchiness to determine pollen source areas of closed-canopy pollen assemblages. Journal of Ecology 82: 89-99.

KÄPILÄ, M. -1984-. Diurnal variation of tree pollen in the air in Finland. Grana 23: 167-176.

LATORRE, F. -1997-. Comparison between phenological and aerobiological patterns of some arboreal species of Mar del Plata (Argentina). Aerobiologia 13: 49- 59.

LATORRE, F., E. J. ROMERO y M. V. MANCINI. -2001-. Representatividad de la vegetación en el espectro de polen de polen atmosférico de la 
ciudad de Mar del Plata (Argentina). Asociación Paleontológica Argentina. Publicación Especial 8. XI Simposio Argentino de Paleobotánica y Palinología, 119-124.

MAJAS,F.D.\&E.J.ROMERO.-1992-.Aeropalynological research in the Northeast of Buenos Aires province, Argentina. Grana 31, 143-156.

MOORE, P. D., J.A. WEBB \& M. E. COLLINSON. -1991-. Pollen analysis. Oxford, Blackwell.

NITIU, D. S. -2003,a-. Annual, daily and diurnal variations of Celtis airborne pollen in La Plata (Argentina). Aerobiología 19 (2), 71-78.

NITIU, D. S. -2003,b-. Análisis polínico anual, diario e intradiurno de Morus en la atmósfera de la ciudad de La Plata, Argentina. XII Simposio Argentino de Paleobotánica y Palinología. Buenos Aires. Libro de resúmenes, 18.

NITIU, D. S., A. C. MALLO \& E. J. ROMERO. -2003-. Quantitative aeropalynology in theatmosphere of Buenos Aires city, Argentina. Aerobiología 19(1): 1-10.

NITIU, D. S. -2006-. Aeropalynologic análisis of La Plata city (Argentina) during a 3-years period. Aerobiologia 22: 79-87.

NITIU, D. S. -2008-. Estudio aeropalinológico de la ciudad de La Plata. Tesis Doctoral 888. Biblioteca Florentino Ameghino. Facultad de Ciencias Naturales y Museo. UNLP.

NITIU, D. S. \& A. C. MALLO. -2002-. Incidence of allergenic pollen of Acer spp., Fraxinus spp. and Platanus spp. in the city of La Plata, Argentina: preliminary results. Aerobiología 18: 65-71.

NITIU, D. S. \& E. J. ROMERO. -2001-. Contenido polínico de la atmósfera de la ciudad de la Plata, Argentina. Polen 11: 79-85.

NITIU, D. S. \& E. J. ROMERO. -2002-. Caracterización aeropalinológica de la atmósfera de la ciudad de la Plata. Vinculación con alergias respiratorias. Boletín de la Sociedad Argentina de Botánica. 37(1-2): 79-85.

O'ROURKE, M. K. -1990-. Pollen reentrainment: contributions to the pollen rain in an arid environment. Grana 29: 147-152.

PÉREZ, C. F., J. M. GARDIOL \& M. M. PAEZ. -2001-. Difusión atmosférica de polen en el sistema urbano-rural de la ciudad de Mar del Plata (Argentina), en los tres últimos meses del año 1995. Polen 11: 87-89.

PLADALMAU, J. M. -1961-. Polen. Ultraestructura y características de los granos de polen. Precisiones morfológicas sobre el polen de especies recolectadas en el NE de España. - Tall. Gráf. D.C.P., Gerona.

SPIEKSMA, F. TH. M., B. H. NIKKELS \& S. BOTTEMA-1994-. Relationship between pollen depositation and airborne pollen concentration. Rev. Paleobot. Palynol. 82: 141-145.

SOLOMON, W. R \& DURHAM, O. C. -1967-. Pollen and Plants that produce them. In J.M. SHELDOM, R.G. LOVELL \& K.P. MATEWS (eds). An annual of Clinical Allergy. Cap. 16, W.B. Sauders Co., Philadelphia.

TRIGO, M. M, M. RECIO, F. J. TORO \& B. CABEZUDO. -1997-. Intradiurnal fluctuations an airborne pollen in Málaga (S. Spain): A quantitative method. Grana 36: 39-43. 
